\title{
An attempt to measure cycloidal rotor fan in a rectangular duct
}

\author{
Tomasz Staśko ${ }^{1 *}$, Mirosław Majkut ${ }^{1}$, Stawomir Dykas ${ }^{1}$, and Krystian Smołka ${ }^{1}$ \\ ${ }^{1}$ A Department of Power Engineering And Turbomachinery, Silesian University of Technology, \\ Gliwice, Poland
}

\begin{abstract}
A fan with cycloidal rotor (CRF) becomes a popular idea in wide application such as aviation, HVAC (heat, ventilation and air conditioning) or marine propeller systems. This is due to advantages such as direct control of the flow direction, larger flow rates than in a conventional machines without cycloidal control. In the presented article, velocity fields of CRF placed in a rectangular channel was measured, using Laser Doppler Anemomentry (LDA) method and thermoanemometric probe (TA).
\end{abstract}

\section{Introduction}

Although the cycloidal rotor was invented more than a century ago [1], it is not widely used nowadays. Mostly it is used as a propulsion of small unmanned aerial vehicles in aviation, in maritime its variant in the form of a cycloidal propeller is used for maneuvering the ship [210]. Thanks to its advantages, such as, the possibility of a quick change of the flow direction [9-11], higher flow efficiency than in the case of machines without cycloidal regulation and the possibility of using a Gurney flap (for fans) [12]. It can also be used for HVAC. The article presents experimental measurements of the speed profiles of a radial fan with a cycloidal rotor in a rectangular duct. The aim of the research was also to determine whether the measurement of Laser Doppler Anemomentry (LDA) can be considered as a benchmark. . For this purpose, it was compared with measurements made with a thermoaneometric probe under the same flow conditions.

\section{Subject of investigation}

The object of measurements was a four-blade radial fan with a cycloidal gear. The blades had a chord of $c=50 \mathrm{~mm}$ and the asymmetric CLARK Y was chosen as the profile. The rotor diameter was $d=140 \mathrm{~mm}$, and the total angle of deflection $\alpha_{0}$ was controlled by a mechanical cycloidal gear.[10,11]

\footnotetext{
* Corresponding author: tomasz.stasko@polsl.pl
} 


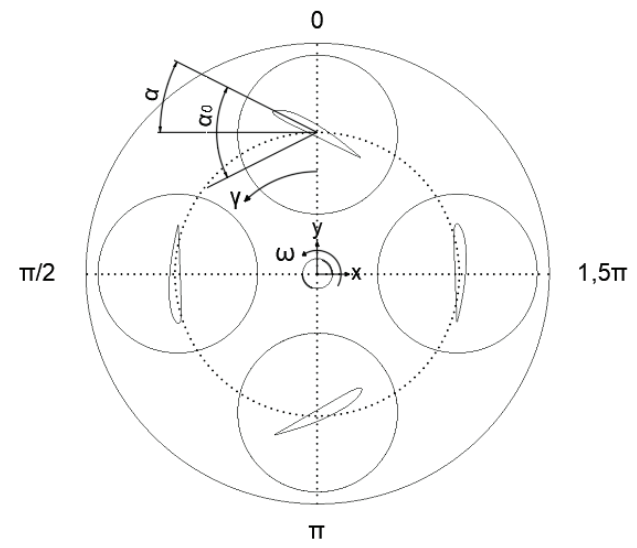

Fig. 1. Schematic drawing of a cycloidal rotor (CRF)

Fig . 1 shows analyzed cycloidal rotor in the starting position. Thanks to the use of a cycloidal gear, the angle $\alpha$ changes with the rotation of the rotor. The blade from the $\gamma=0$ position with a positive deflection angle $\alpha$, passing to the $\gamma=\pi / 2$ position, resets this angle to $\alpha=0^{\circ}$, so that in the opposite position $(\gamma=\pi)$ to the initial position, the same angle $\alpha$ is obtained only with a negative value. Then, in the $\gamma=1,5 \pi$ position, the deflection angle $\alpha$ is again reset to zero (the angle should be the same as in $\gamma=\pi / 2$ ) and finally the blade returns to its original position with a positive deflection angle. Each blade rotates around a middle point of camber line of an airfoil.
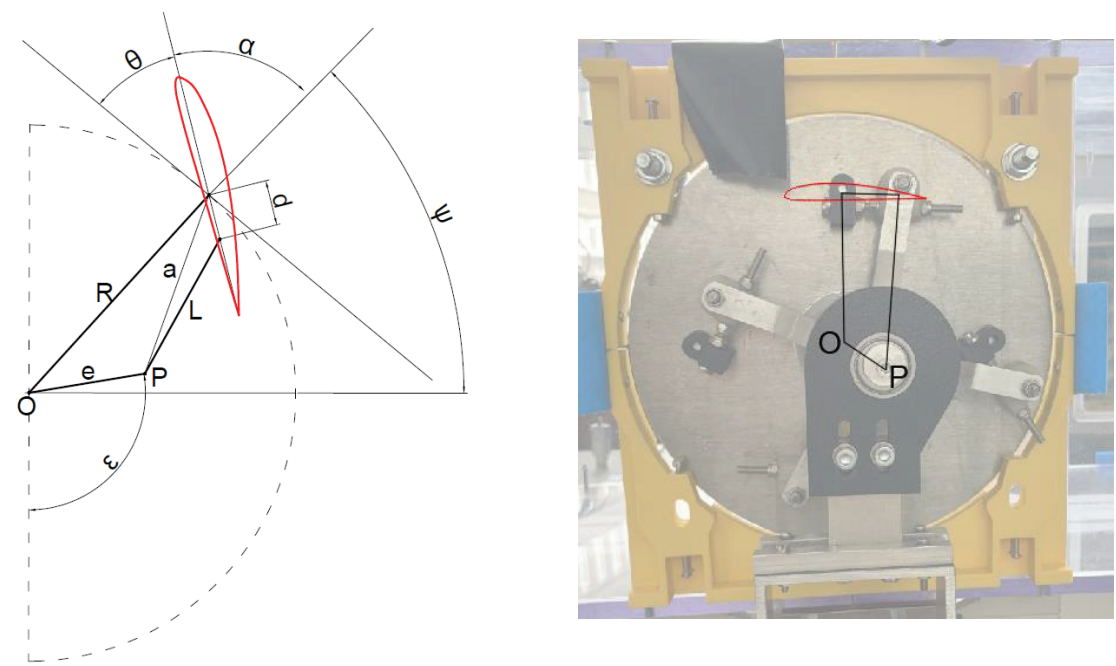

Fig 2. Cycloidal regulation with its geometrical parameters

Fig. 2 shows the cycloidal gear from the analyzed CRF with its characteristic values marked. Using ready-made equations, a program was written that allowed to determine the control parameters for optimal changes in angles and for plotting the corresponding cycloidal functions describing the movement of the blade in a circle. It was based on the following equations:[5] 


$$
\begin{gathered}
a=\sqrt{e^{2}+R^{2}-2 e R \cos \left(\psi+\varepsilon+\frac{\pi}{2}\right)} \\
L=\sqrt{a^{2}+d^{2}-2 a d \cos \left(\alpha_{2}\right)} \\
\theta=\frac{\pi}{2}-\sin ^{-1}\left[\frac{e}{a} \cos (\psi+\varepsilon)\right]-\cos ^{-1}\left[\frac{a^{2}+d^{2}-L^{2}}{2 a d}\right]
\end{gathered}
$$

The first formula (1) determines the length of the segment $a$ between the axis of regulation $P$ (point $P$ is the result of a vertical and horizontal shift of the regulation - this shift is defined as the $\varepsilon$ angle). The second one (2) determines the length of the tendon $L$ from the point $P$ to the point of attachment of the blade (in this case it is its center). The third (3) formula determines the relationship between the angle of the blade $\theta$ and its location on the circle $\psi$. The other geometric quantities are: $R$ - rotor diameter, $e$ - distance shifted by the $\varepsilon$ angle by which the regulation was shifted and $d$ - distance of the strand hook $\mathrm{L}$ from the center of the blade. Using the above-mentioned program, the predicted real cycloid function, which was a sixth degree polynomial function, was plotted and compared with the ideal cycloid function, which was a cosine function shifted by the analyzed angle. The rotor blades and its adjustments were set in accordance with the determined parameters, and on this basis, the actual cycloidal function was obtained, characteristic for this setting.

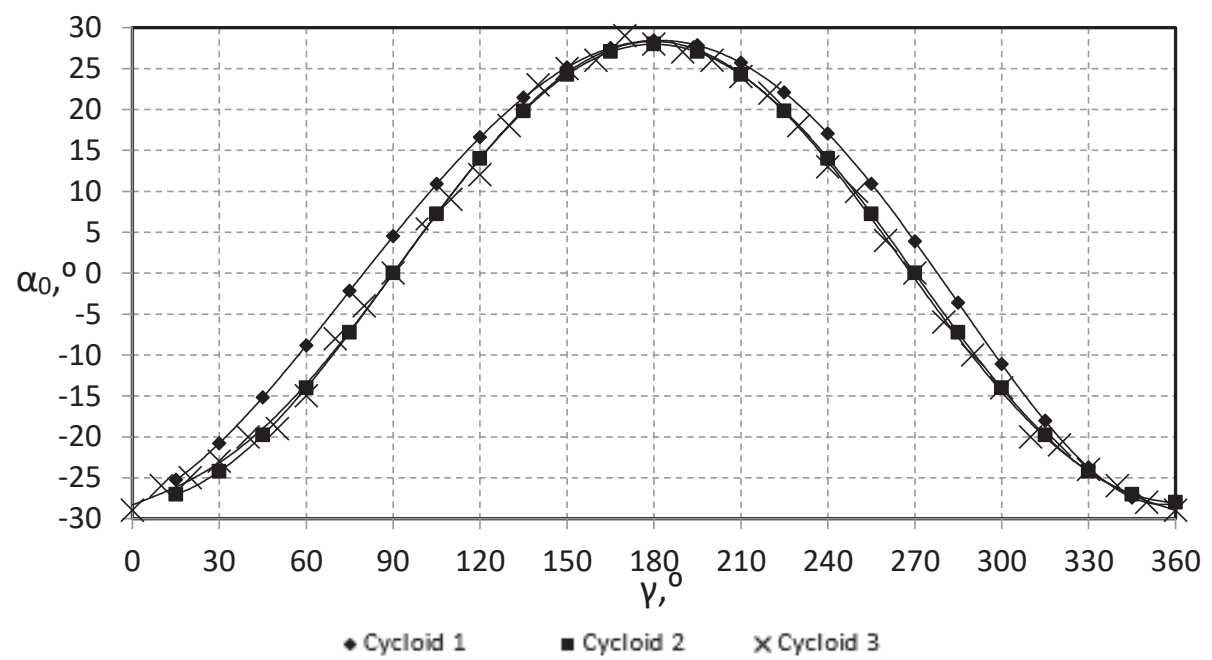

Fig 3. Comparison of cycloidal functions (Cycloid 1 - ideal cycloidal function, Cycloid 2 - predicted cycloid function, Cycloid 3 - actual cycloid function)

Fig. 3 shows a comparison of 3 cycloids. For all cases, the deflection angle $\alpha_{0}$ was checked depending on the position of the blade on the circle $\gamma$. Cycloid 1 is an ideal cyclic function describing the change in the angle of the rotor blade from -30 to $+30^{\circ}$. Cycloid 2 is a function determined using a program written for this purpose, and on the basis of this function, which is a polynomial function, the geometric parameters of the cyclorotor for the analyzed range of angles were determined. Cycloid 3 is a function determined on the basis of measurements of the actual angles of the rotor blades. It can be seen that the predicted Cycloid 2 practically coincided with the real Cycloid 3, which means that the machine with the adjustment was assembled correctly. Both of the above-mentioned functions slightly differ from the ideal. 


\section{Measurements}

The stand consisted of a rectangular duct with a fan with a cycloidal rotor. The channel was made of solid polycarbonate plates, its length was $4 \mathrm{~m}$, and the diameter was $250 \times 250 \mathrm{~mm}$. To prevent unfavorable phenomena at the fan inlet, the CRF was placed in the middle of the duct. The cycloidal fan was measured in a closed tunnel using two methods: measuring the velocity profiles with TA and LDA. [13-15] The blade pitch angle was set to $\alpha=28^{\circ}$ and the rotor speed was set to $1000 \mathrm{rpm}$.

a)

b)
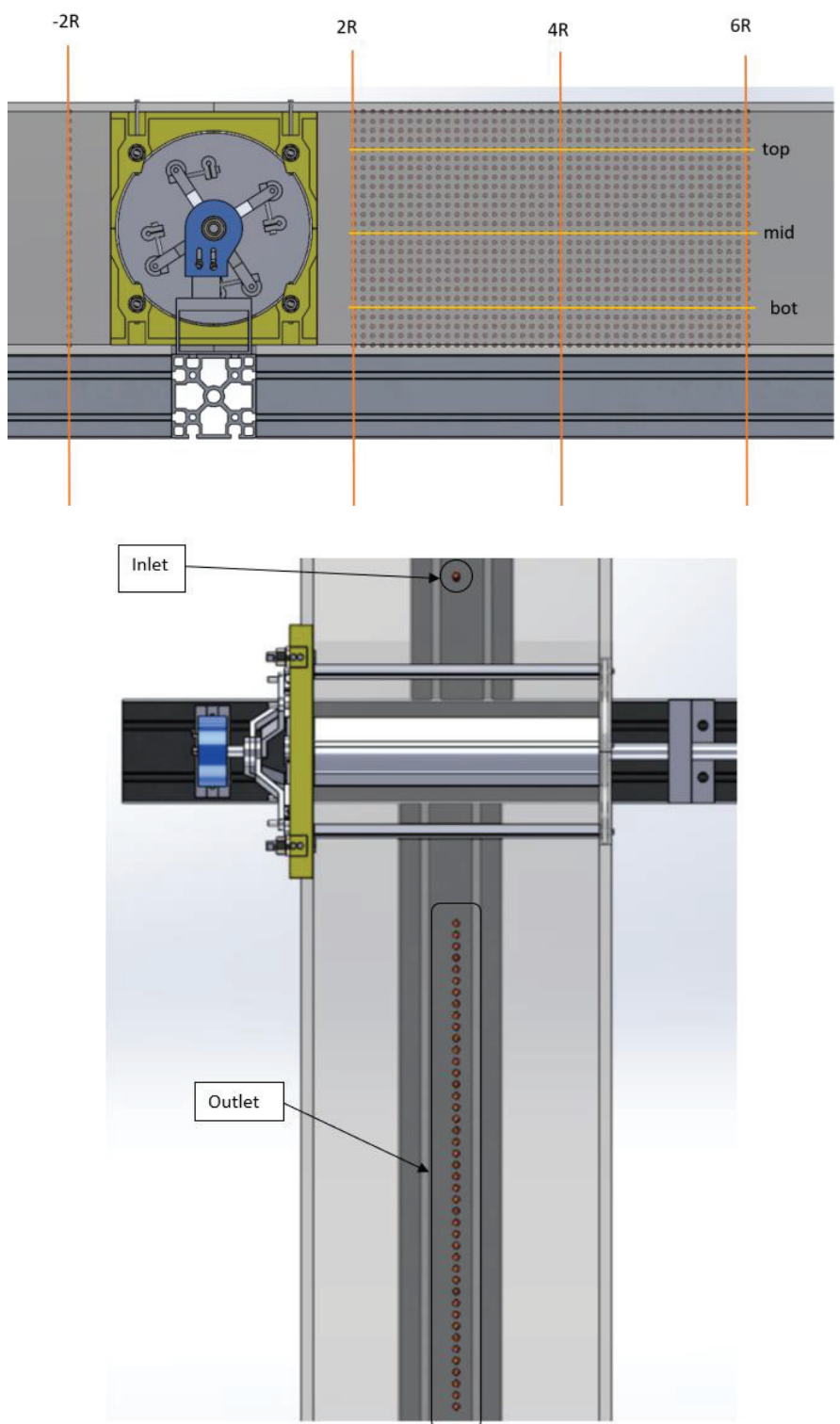

Fig. 4. Measurement areas of CRF a) plane locations b) location of measurement grid in channel 
The example above (Fig. 4) shows measurement area with marked measurements points. In Figure $4 a$, horizontal (top, mid, bot) and vertical $(-2 R, 2 R, 4 R$ and $6 R)$ measurement planes were showed. The horizontal planes were located to coincide with the rotor diameter. The top plane was located at the height $+\mathrm{R}$ from the rotor center, the lower plane was located at the same height as $-\mathrm{R}$, and the middle plane along the rotor axis. The vertical planes were located at a distance $\mathrm{R}$ (radius) from the rotor axis, so the inlet plane was 2 times $\mathrm{R}$ from the axis, and the outlet plane was 2 to $6 \mathrm{R}$. The measuring grid is located in the middle of the canal (Fig. 4b). Using the created mesh, a series of measurements were made using LDA and TA. The aim of the measurements was to determine the velocity profiles for the analyzed planes, to compare the results of both methods and to define the LDA method as the reference method.

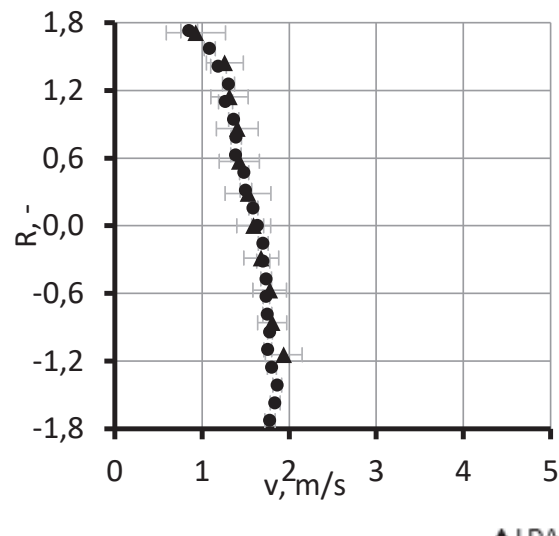

a) Velocity profile in the inlet $(-2 \mathrm{R})$

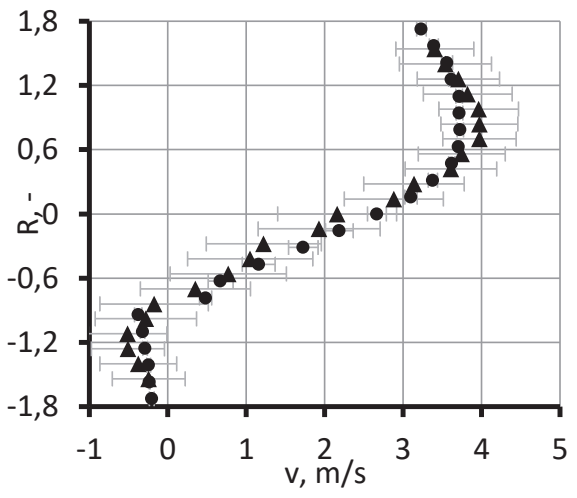

$\triangle$ LDA

c) Velocity profile in the outlet (4R)

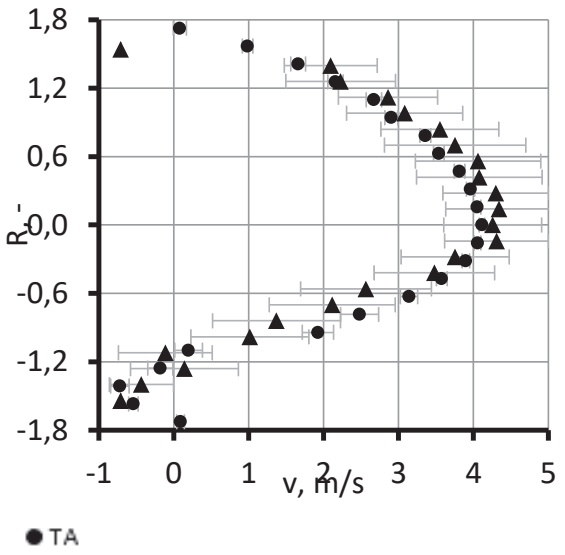

b) Velocity profile in the outlet (2R)

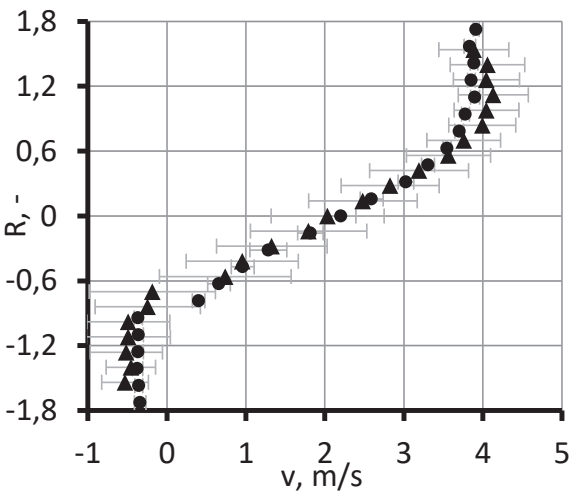

d) Velocity profile in the outlet (6R)

Fig. 5. Comparison of the velocity profiles across the channel. Velocity profiles measured a) on inlet at $2 R \mathrm{~b}$ ) on outlet at $2 R \mathrm{c}$ ) on outlet at $4 R \mathrm{~d}$ ) on outlet at $6 R$

Fig. 5 shows the velocity profiles determined with LDA and TA for different channel cross sections with standard deviation bars for each method. It can be seen that for the inlet profile Fig. 5a the velocity profile is not vertical. Increased velocity values at the bottom of the plane suggest that the rotor draws the stream more from the bottom than from entire width of the 
channel. In the case of the $2 R$ outlet, (Fig $5 \mathrm{~b}$ ) the velocity profile is already developed and its face is practically in the axis of the rotor. During the measurements, the formation of significant turbulence in the vicinity of the upper and lower part of the flow was noticed, as indicated by negative velocity values. In the case of planes $4 R$ and $6 R$, (Fig $5 \mathrm{c}, \mathrm{d}$ ) the velocity profiles are very similar to each other. As the flow moves away from the rotor, it is directed more and more towards the main tunnel wall, which may cause it to rebound and slow down slightly. The formation of vortices in the lower part of the test tunnel was also observed for both profiles. Measurement errors were also determined for each velocity profile. It can be seen that for all velocity profiles, the measurements overlap satisfactorily, not exceeding the specified values of the error bars. Volumetric flow equal $0,12 \mathrm{~m}^{3} / \mathrm{s}$ was determined with the use of LDA for inlet and inlet section.

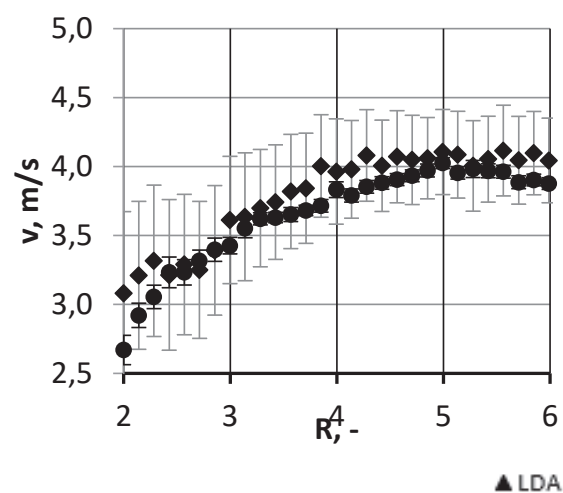

a) Velocity profile in the top plane

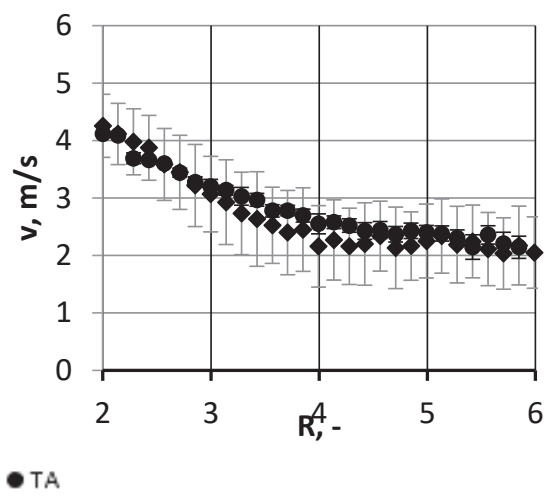

b) Velocity profile in the center plane

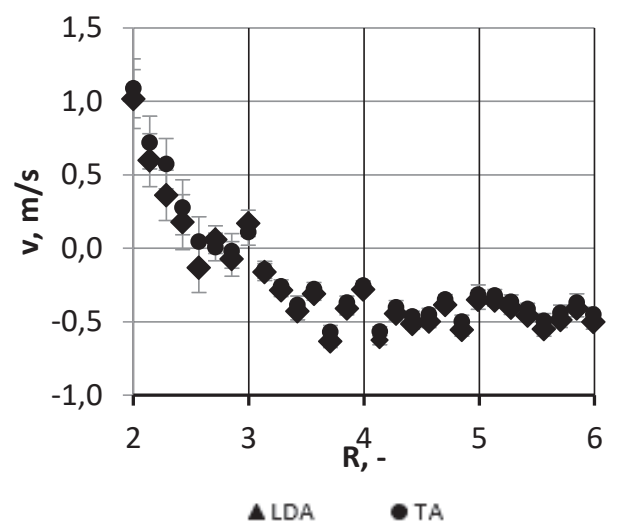

c) Velocity profile in the bottom plane

Fig. 6. Comparison of the velocity profiles across the channel. Velocity profiles measured a) on top plane b) on center plane c) on bottom plane

Fig. 6 shows the velocity profiles determined with LDA and TA for the cross section along the duct at different heights. The plane described as "top" is situated at the height of the upper edge of the rotor at a distance $R$ from its center and extends from $2 R$ to $6 R$. The "center" distance is measured along the axis of the rotor (shaft) to the same length as the "top" part. The "bottom" plane is measured at the distance $-R$ and has the same length as the previous ones. In the case of the "top" plane, an increase in speed can be seen depending on the length 
of the exit section. The farther from the rotor, the flow velocity in the layer increases. In the "center" plane, it can be observed a decrease in the velocity value, which is associated with a change in the flow direction - the flow begins to go up in the tunnel. In the "bottom", the velocity drops significantly to its negative values, caused by formation of vortices. As in the previous case, the compliance of the LDA and TA measurements can be considered satisfactory. As can be seen, the LDA measurement can be considered as a benchmark for further measurements and numerical calculations.

\section{Conclusions}

The article describes a methods of measurements and the results of a fan with a cycloidal rotor placed in a rectangular duct. The analyzed fan had a 4-blade impeller with a diameter $d$ of $140 \mathrm{~mm}$, with blades with an asymmetrical CLARK Y profile. Measurements were carried out using two methods: one non-intrusive which was Laser Doppler Anemometry (LDA) and second intrusive - thermoanemometric probe (TA). The aim of the measurements was to determine velocity profiles for various cross-sectional areas and planes and to validate both of the measurement methods. For the given measurement regions, velocity profiles were determined along with the determination of the measurement error for both measurement methods. All the results obtained overlap to a satisfactory degree, which allows to assume that the LDA method will be the reference method and used in future measurements due to the previously obtained validation, not affecting the measurement and the possibility of visualizing the obtained results.

\section{References}

1. M. Benedict, Fundamental understanding of cycloidal - rotor concept for micro air vehicle applications. (2010).

2. J. Augusto, L. Monteiro, J. Páscoa, C. Xisto, Aerodynamic optimization of cyclorotors. Aircraft Engineering and Aerospace Technology. (2016).

3. C. Xisto, J. Páscoa, M. Trancossi, Geometrical Parameters Influencing the Aerodynamic Efficiency of a Small-Scale Self-Pitch High Solidity VAWT, April (2016), Journal of Solar Energy Engineering 138(3):pp.10

4. C. Xisto, J. Leger, J. Páscoa, L. Gagnon, P. Masarati, D. Angeli, A. Dumas, Parametric Analysis of a Large - scale Cycloidal Rotor in Hovering Conditions, July (2016), Journal of Aerospace Engineering 30(1).

5. A. Andrisani, D. Angeli, A. Dumas, Optimal pitching schedules for a cycloidal rotor in hovering August (2016), Aircraft Engineering and Aerospace Technology 88(5)

6. E. Shrestha, D. Yeo, M. Benedict, I. Chopra, Development of a Meso-scale Cycloidalrotor Aircraft for Micro Air Vehicle Application, May (2017), International Journal of Micro Air Vehicles 9(3)

7. M. Rami and J. Pascoa, "Numerical Analysis of a Cycloidal Rotor under Diverse Operating Conditions and Altitudes," SAE Technical Paper 2019-01-1872, (2019), doi:10.4271/2019-01-1872.

8. M. Benedict, M. Ramasamy, I. Chopra, Improving the Aerodynamic Performance of Micro-Air-Vehicle-Scale Cycloidal Rotor: An Experimental Approach, July (2010), Journal of Aircraft 47(4):1117-1125 
9. E. Shrestha, D. Yeo, M. Benedict, I. Chopra, Development of a Meso-scale Cycloidalrotor Aircraft for Micro Air Vehicle Application, May (2017), International Journal of Micro Air Vehicles 9(3):

10. S. Dykas S, M. Majkut, K. Smołka, M. Strozik, T. Chmielniak, T. Staśko, Numerical and Experimental Investigation of the Fan with Cycloidal Rotor, Mechanics and Mechanical Engineering Vol. 22, No. 2 (2018) 447-454

11. T. Staśko, S. Dykas, M. Majkut, K. Smołka, An attempt to evaluate the cycloidal rotor fan performance, Open Journal of Fluid Dynamics, (2019), 9, 292-30

12. P. Wiśniewski, F. Balduzzi, Z. Buliński, A. Bianchini, A Numerical Analysis on the Effectiveness of Gurney Flaps as Power Augumentation Devices for Airfoils Subject to a Continuous Variation of the Angle of Attack by Use od Full and Surrogate Models, Energies, vol:13,(2020)

13. C. Xisto, J. Páscoa, J. Leger, Cycloidal Rotor Propulsion System with Plasma Enhanced Aerodynamics. In ASME 2014 International Mechanical Engineering Congress \& Exposition; November 14-20, (2014); Montreal, Canada.

14. F. Heitmann, M. Juling, J. Steinbock, Performance of the LDA Volumetric Flow Rate Standard Under Severely Disturbed Flow Conditions, Flow Measurement and Instrumentation, Volume 74, (2020), 101756, ISSN 0955-5986,

15. Y. Han, S.Q. Yang, N. Dharmasiri, M. Sivakumar, Effects of sample size and concentration of seeding in LDA measurements on the velocity bias in open channel flow, Flow Measurement and Instrumentation, Volume 38, (2014), Pages 9297, ISSN 0955-5986, 